\title{
Metilprednisolona no trauma raquimedular: revisão sistemática
}

\author{
Methylprednisolone in spinal trauma: a systematic review \\ Metilprednisolona en el trauma espinal: una revisión \\ sistemática
}

\author{
Leandro Januário de Lima1, Francisco José Ferreira Filho ${ }^{1}$, Nathã \\ Romullo Alves Barbosa², Maria do Carmo Andrade Duarte de Farias 3
}

1.Graduando em Medicina, Universidade Federal de Campina Grande (UFCG), Campus Cajazeiras, Cajazeiras-PB, Brasil. Bolsista do Conselho Nacional de Desenvolvimento Científico e Tecnológico (CNPq). 2.Graduando em Medicina, Universidade Federal de Campina Grande (UFCG), Campus Cajazeiras, Cajazeiras-PB, Brasil.

3.Enfermeira. Pós-Doutora em Ciências da Saúde pela Faculdade de Medicina do ABC (FMABC). Professora Titular na Universidade Federal de Campina Grande (UFCG), Campus Cajazeiras, Cajazeiras-PB, Brasil.

\section{Resumo}

Introdução. A metilprednisolona é um tratamento farmacológico para lesões medulares traumáticas agudas, cuja recomendação do uso permanece controversa. Objetivo. Descrever as evidências do uso de metilprednisolona nos pacientes com traumatismo raquimedular. Método. Trata-se de uma revisão sistemática da literatura. Pesquisou-se nas bases Lilacs e Medline estudos clínicos randomizados de pacientes que receberam metilprednisolona no braço intervenção, em comparação com placebo ou outra droga, com início do tratamento em até 48 horas do diagnóstico do trauma. Não houve restrição de idioma ou data de publicação. Os desfechos primários avaliados foram mortalidade, função motora, sensibilidade superficial e/ou profunda, enquanto os secundários foram efeitos adversos, qualidade de vida e independência funcional. Os estudos foram avaliados quanto ao viés pelo score de Jadad, enquanto a evidência foi classificada conforme os níveis da Universidade de Oxford. Os resultados foram sumarizados de forma qualitativa. Resultados. Cinco publicações foram incluídas na análise, todas no nível um de evidência para estudos de benefício de tratamento, com 1092 pacientes randomizados. $40 \%$ dos estudos atingiram score de boa qualidade, com o mascaramento e descrição das perdas de segmento foram os que acarretaram mais rebaixamentos de pontuação. Conclusão. Não recomendamos o uso de metilprednisolona, face a ausência de benefício de melhora significativa da função motora e sensibilidade, além de não reduzir mortalidade. A independência funcional também não apresentou melhora, enquanto a qualidade de vida sequer foi avaliada nos estudos incluídos. O uso da droga também se associou à elevação de efeitos adversos.

Unitermos. Metilprednisolona; Traumatismos da Medula Espinhal; Traumatismos do Sistema Nervoso; Tratamento Farmacológico; Neurocirurgia; Cuidados Críticos

\footnotetext{
Abstract

Introduction. Methylprednisolone is a pharmacological treatment for acute traumatic spinal cord injuries, the use of which remains controversial. Objective. To describe the evidence of the use of methylprednisolone in patients with spinal trauma. Method. This is a systematic review of the literature. Randomized clinical studies of patients who received methylprednisolone in the intervention arm, compared with placebo or another drug, were investigated in the Lilacs and Medline databases, compared to placebo or another drug, starting treatment within 48 hours of the trauma diagnosis. There was no language restriction or publication date. The primary outcomes assessed were mortality, motor function, superficial and / or deep sensitivity, while the secondary outcomes were adverse effects, quality of life and functional independence. The studies were assessed for bias by the Jadad score, while the evidence was classified according to the levels of the University of Oxford. The results were summarized qualitatively. Results. Five publications were included in the analysis, all at level one of evidence for treatment benefit studies, with 1092 randomized patients. $40 \%$ of the
} 
studies achieved good quality scores, with the masking and description of the segment losses being the ones that caused the most score downgrades. Conclusion. Ee do not recommend the use of methylprednisolone, due to the lack of significant improvement in motor function and sensitivity, in addition to not reducing mortality. Functional independence also did not improve, while quality of life was not even assessed in the included studies. The use of the drug was also associated with increased adverse effects.

Keywords. Methylprednisolone; Spinal Cord Injuries; Trauma, Nervous System; Drug Therapy; Neurosurgery; Critical Care

\section{Resumen}

Introducción. La metilprednisolona es un tratamiento farmacológico de las lesiones traumáticas agudas de la médula espinal, cuyo uso sigue siendo controvertido. Objetivo. Describir la evidencia del uso de metilprednisolona en pacientes con trauma espinal. Método. Se trata de una revisión sistemática de la literatura. Los estudios clínicos aleatorizados de pacientes que recibieron metilprednisolona en el brazo de intervención, en comparación con placebo u otro fármaco, se investigaron en las bases de datos Lilacs y Medline, en comparación con placebo u otro fármaco, comenzando el tratamiento dentro de las 48 horas posteriores al diagnóstico del trauma. No hubo restricciones de idioma ni fecha de publicación. Los resultados primarios evaluados fueron mortalidad, función motora, sensibilidad superficial y/o profunda, mientras que los resultados secundarios fueron efectos adversos, calidad de vida e independencia funcional. Se evaluó el sesgo de los estudios mediante la puntuación de Jadad, mientras que la evidencia se clasificó según los niveles de la Universidad de Oxford. Los resultados se resumieron cualitativamente. Resultados. Se incluyeron cinco publicaciones en el análisis, todas en el nivel uno de evidencia para estudios de beneficio del tratamiento, con 1092 pacientes aleatorizados. El 40\% de los estudios obtuvieron puntajes de buena calidad, siendo el enmascaramiento y descripción de las pérdidas de segmento las que causaron más bajadas de puntaje. Conclusión. No recomendamos el uso de metilprednisolona, debido a la falta de mejoría significativa en la función motora y la sensibilidad, además de no reducir la mortalidad. La independencia funcional tampoco mejoró, mientras que la calidad de vida ni siquiera se evaluó en los estudios incluidos. El uso de la droga también se asoció con un aumento de los efectos adversos.

Palabras clave. Metilprednisolona; Traumatismos de la Médula Espinal; Traumatismos del Sistema Nervioso; Quimioterapia; Neurocirugia; Cuidados Críticos

Trabalho realizado na Universidade Federal de Campina Grande (UFCG), Cajazeiras-PB, Brasil.

\section{INTRODUÇÃO}

\section{A lesão medular traumática aguda é uma condição que} causa impacto pessoal e social negativos sobre os indivíduos. A injúria primária, seja contusa ou penetrante, é seguida de alterações imediatas como hemorragia e redução do aporte de adenosina trifosfato, que culminam em ativação microglial e apoptose celular ${ }^{1}$. 
A sobrevivência dos pacientes com trauma medular que evoluem com comprometimento cardiorrespiratório é menor naqueles do sexo feminino, casos com secções completas, lesão localizada na coluna cervical, indivíduos com depressão e que sofrem de disreflexia autonômica².

Nos Estados Unidos, a taxa de morbidade de 54 casos em um milhão de habitantes, registrada em 2012, foi pouco superior aos 53 casos de 1993, mas reflete um número absoluto de casos crescente face ao crescimento populacional do período. O cenário epidemiológico aponta haver uma redução das taxas entre os pacientes mais jovens simultâneo à elevação da incidência entre os mais idosos, associada ao aumento das quedas como etiologia traumática, além de manutenção de alta mortalidade intrahospitalar, a despeito do emprego de recursos de alta complexidade de assistência ${ }^{3}$.

Além da abordagem protocolar aos traumas, o uso da metilprednisolona foi encorajado no tratamento farmacológico das injúrias medulares mecânicas como uma medida específica. O objetivo era exercer um efeito neuroprotetor contra as lesões secundárias, aquelas que se desenvolvem de segundos a anos após a lesão inicial, ainda que o mecanismo de ação neste caso não seja muito conhecido ${ }^{4}$. O potencial efeito neuroprotetor pela redução da cascata inflamatória local é associado à redução das perdas motoras, embora ainda não seja recomendado globalmente nas diretrizes clínicas. A administração de riluzol, o uso de transplante celular ou administração de fatores de 
crescimento celular são terapêuticas emergentes cujos efeitos ainda não são plenamente conhecidos ${ }^{1}$.

Diante de um cenário de necessidade de novos estudos clínicos para testes de terapias farmacológicas no trauma raquimedular, 0 emprego de altas doses de metilprednisolona, administradas até oito horas após a lesão medular, se mostrou promissor em ensaios randomizados de fase três, ainda que sob questionamentos dos seus resultados sobre o uso em todos os pacientes ${ }^{5}$.

Neste sentido, realizou-se este estudo com o objetivo de descrever as evidências do uso de metilprednisolona nos pacientes com traumatismo raquimedular. Como pergunta de partida, elencou-se: em pacientes atendidos com traumatismo da medula espinhal, independente da síndrome clínica medular, quais os efeitos da administração de metilprednisolona sobre a neuroproteção e recuperação?

\section{MÉTODO}

\section{Tipo de Estudo}

Trata-se de uma revisão sistemática de ensaios clínicos randomizados (ECRs) sobre o efeito neuroprotetor da metilprednisolona nos pacientes com traumatismo raquimedular, com metodologia inspirada no manual de revisões sistemáticas do Ministério da Saúde brasileiro ${ }^{6}$. Não houve submissão à plataforma PROSPERO de registro de revisões sistemáticas, mas o relato segue as diretrizes dos 
principais itens para relato de revisões sistemáticas e metanálise - protocolo PRISMA 7 .

\section{Local do Estudo}

Esta revisão foi realizada no âmbito de Liga de Medicina Baseada em Evidências (LAMEBE), da Universidade Federal de Campina Grande (UFCG). A busca nas bases de dados foi realizada em 15 de abril de 2020.

\section{Critérios de Inclusão dos Estudos}

Tipos de Estudos

Foram incluídos apenas estudos de intervenção, do tipo ensaios clínicos randomizados, independente do desenho do estudo ou técnica de randomização. Não foram incluídos protocolos de ensaios clínicos, estudos observacionais quase-experimentais ou relatos de resultados parciais de estudos em andamento.

Tipos de Participantes

Considerou-se para inclusão os estudos com pacientes adultos ou infantis com diagnóstico clínico ou radiológico de lesão traumática da medula espinhal, excluindo-se aqueles cuja população incluída fosse portadora de traumatismo multissistêmico, cujo uso da intervenção-alvo pelos pacientes não se desse em virtude da lesão medular, e sim por qualquer outra indicação. 
Tipo de intervenção

Uso de metilpredinisolona endovenosa com início em até 48 horas do diagnóstico do trauma raquimedular. Aceitou-se eventual estudo em que a intervenção para o trauma raquimedular foi combinada, desde que o segundo tratamento também tenha sido oferecido ao grupo controle. A comparação se deu entre a intervenção e placebo, tratamento padrão ou outro fármaco.

\section{Processo de busca dos estudos}

Realizou-se a busca pelos estudos em abril de 2020, utilizando duas das bases de dados essenciais elencadas pelo manual do Ministério da Saúde ${ }^{6}$, a saber: LILACS (Literatura Latino-Americana e do Caribe em Ciências da Saúde), via Biblioteca Virtual de Saúde, e MEDLINE (Medical Literature Analysis and Retrieval System Online), via PubMed. Não foi aplicada restrição de idioma ou data de publicação. As estratégias de busca (Quadro 1) basearam-se no uso dos descritores padronizados por cada coleção própria para a base, seus sinônimos e códigos para aumentar a sensibilidade. Os termos padronizados na busca para a LILACS foram selecionados no DeCS (Descritores em Ciências da Saúde), enquanto os da MEDLINE são provenientes do MeSH (Medical Subject Headings).

\section{Seleção dos Estudos e Extração de dados}

Dois pesquisadores (LJL e FJFF), independentemente, procederam as fases de elegibilidade e inclusão dos estudos. 
As discordâncias foram passivamente resolvidas pelo julgamento de um terceiro avaliador (NRAB). Os mesmos autores da seleção realizaram a extração dos dados dos estudos incluídos na análise qualitativa.

Quadro 1. Estratégia de busca nas bases de dados.

\begin{tabular}{|l|l|}
\hline Base & Estratégia de Busca \\
\hline LILACS & \#1 (Methylprednisolone OR Metilprednisolona OR \\
D04.210.500.745.432.769.795.539) \\
\#2 ("Methylprednisolone Hemisuccinate" OR "Hemisuccinato de \\
Metilprednisolona" OR "Hemissuccinato de Metilprednisolona" OR "Succinato \\
de Metilprednisolona" OR D04.210.500.745.432.769.795.539.500) \\
\#3 ("Methylprednisolone Acetate" OR "Acetato de Metilprednisolona" OR \\
D04.210.500.745.432.769.795.539.250) \\
\#4 \#1 OR \#2 OR \#3 \\
\#5 ("Spinal Cord Injuries" OR "Traumatismos da Medula Espinhal" OR \\
"Traumatismos de la Médula Espinal" OR "Mielopatia Traumática" OR \\
"Traumatismos da Medula Espinal" OR "Traumas Medulares" OR "Traumas da \\
Medula Espinhal" OR "Traumatismos Medulares" OR "Trauma Raquimedular" \\
OR "Traumatismo Raquimedular" OR C10.228.854.763 OR C10.900.850 OR \\
C26.819) \\
\#6 \#4 AND \#5 \\
\#1 ("Methylprednisolone"[Mesh] OR Metipred OR 6-Methylprednisolone OR "6 \\
Methylprednisolone" OR Urbason OR Medrol) \\
\#2 ("Methylprednisolone Hemisuccinate"[Mesh] OR "Methylprednisolone \\
Acetate"[Mesh]) \\
\#3 \#1 OR \#2 \\
\#4 ("Spinal Cord Injuries"[Mesh] OR "Spinal Cord Trauma" OR "Cord Trauma, \\
Spinal" OR "Cord Traumas, Spinal" OR "Spinal Cord Traumas" OR "Trauma, \\
Spinal Cord" OR "Traumas, Spinal Cord") \\
\#5 \#3 AND \#4
\end{tabular}

Os dados extraídos foram concernentes aos seguintes desfechos primários: mortalidade, função motora, sensibilidade superficial e/ou profunda. Como desfechos secundários, pesquisou-se dados sobre efeitos adversos das drogas, índices de qualidade de vida e scores de independência funcional.

\section{Avaliação Crítica dos Estudos}

O risco de viés das publicações também foi avaliado em duplo-cego pelos pesquisadores (LJL e FJFF) utilizando-se de 
ferramenta padronizada ${ }^{8}$. O nível de evidência foi definido a partir da classificação do Centre for Evidence-Based Medicine da Universidade de Oxford ${ }^{9}$.

\section{Síntese dos Dados}

Realizou-se uma abordagem qualitativa dos estudos incluídos, em virtude da heterogeneidade da mensuração dos desfechos analisados.

\section{RESULTADOS}

\section{Busca nas Bases}

Foram identificados nas bases pesquisadas 825 publicações após à busca nas plataformas pelas fórmulas. Aplicando-se os filtros para limite da pesquisa com seres humanos, estudos clínicos e ensaios clínicos randomizados, 59 artigos se tornaram elegíveis para o estudo, dos quais cinco foram incluídos após a leitura do texto integral. Os cinco artigos englobam três estudos diferentes, com seguimento máximo dos pacientes pelo período de um ano. O fluxograma de seleção PRISMA encontra-se na Figura 1.

\section{Estudos Incluídos}

Os cinco trabalhos incluídos englobam os resultados de três estudos, com seguimentos instantâneos e de até um ano. As publicações compreenderam o período entre 1990 e 2000, com dois dos três estudos realizados nos Estados 
Unidos da América e um na República da França. Apenas o estudo francês foi realizado em um único centro (Quadro 2).

Figura 1. Processo de elegibilidade e seleção dos estudos.

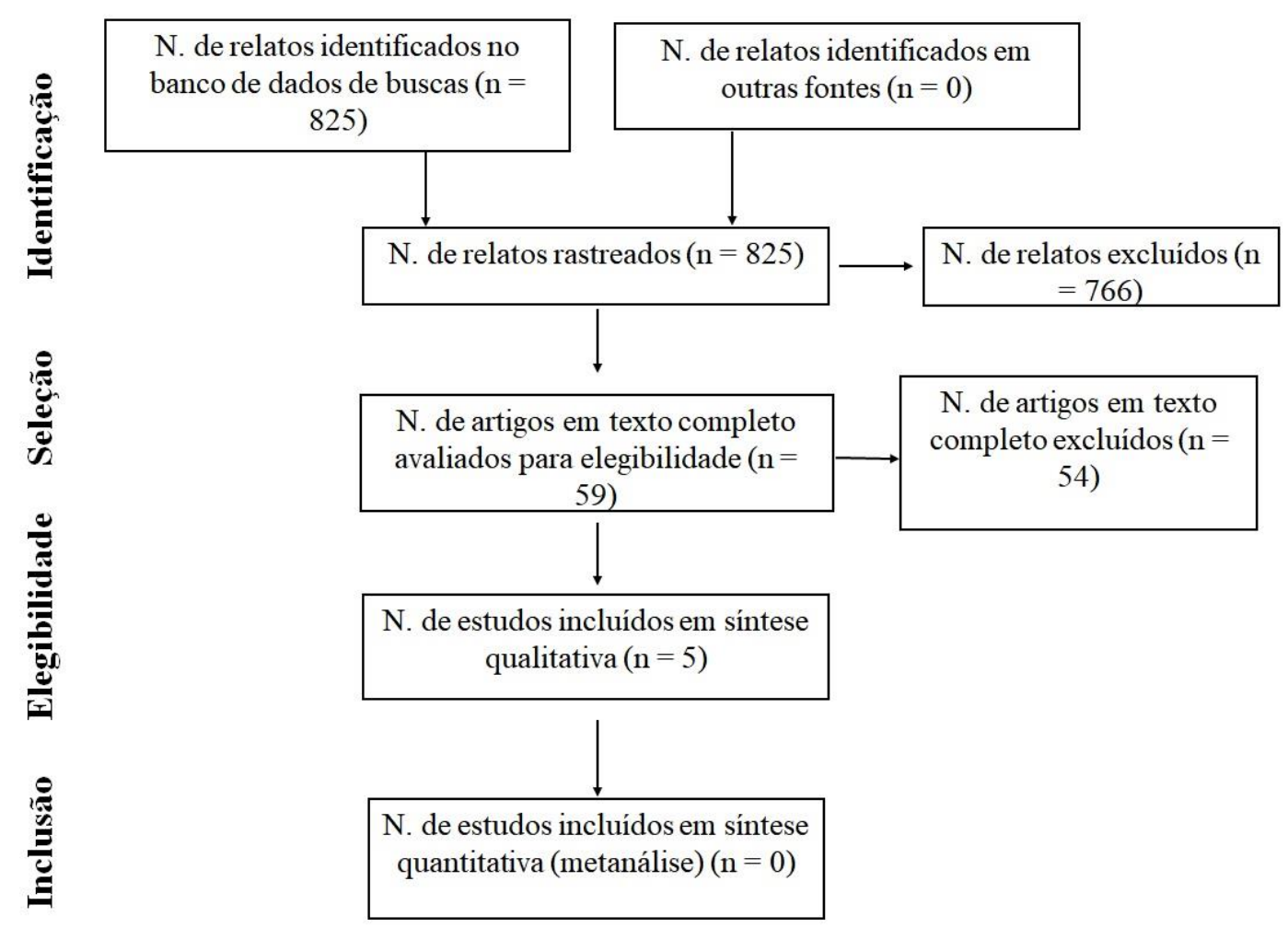

As publicações centraram-se na divulgação dos resultados dos estudos nacionais sobre trauma raquimedular dos Estados Unidos: second National Acute Spinal Cord Injury Study ${ }^{10,11}$ (NASCIS II) e third National Acute Spinal Cord Injury Study ${ }^{12,13}$ (NASCIS III). Estes estudos compõem $80 \%$ dos artigos incluídos. Cada um destes estudos teve duas publicações, com seguimentos de curto e médio prazo $^{10,12}$ e de longo prazo (um ano) ${ }^{11,13}$. A decisão de incluir ambos os seguimentos foi comum aos três avaliadores do 
processo de elegibilidade, o que aumenta o alcance dos resultados pelo segmento mais amplo.

Quadro 2. Matriz de síntese dos estudos incluídos.

\begin{tabular}{|c|c|c|c|c|}
\hline $\begin{array}{c}\text { Autor } \\
\text { Principal }\end{array}$ & $\begin{array}{c}\text { Ano de } \\
\text { publicação }\end{array}$ & Intervenção & $\begin{array}{c}\text { Número } \\
\text { de } \\
\text { centros }\end{array}$ & $\begin{array}{c}\text { Local } \\
\text { do } \\
\text { estudo }\end{array}$ \\
\hline Bracken $^{10}$ & 1990 & $\begin{array}{l}\text { Grupo } 1 \text { ( } n=162 \text { ): Metilprednisolona } 30 \\
\text { mg/kg em bolus, seguido de } 5,4 \mathrm{mg} / \mathrm{kg} / \mathrm{h} \\
\text { durante } 23 \text { horas. } \\
\text { Grupo } 2(\mathrm{n}=154): \text { Naloxona } 5,4 \mathrm{mg} / \mathrm{kg} \\
\text { em bolus, seguida de } 4,0 \mathrm{mg} / \mathrm{kg} / \mathrm{h} \\
\text { durante } 23 \text { horas. } \\
\text { Grupo } 3 \text { ( } \mathrm{n}=171 \text { ): administração do } \\
\text { placebo nos mesmos regimes } \\
\text { posológicos. }\end{array}$ & 10 & EUA \\
\hline Bracken $^{11}$ & 1992 & $\begin{array}{l}\text { Grupo } 1 \text { ( } \mathrm{n}=162): \text { Metilprednisolona } 30 \\
\mathrm{mg} / \mathrm{kg} \text { em bolus, seguido de } 5,4 \mathrm{mg} / \mathrm{kg} / \mathrm{h} \\
\text { durante } 23 \text { horas. } \\
\text { Grupo } 2(\mathrm{n}=154): \text { Naloxona } 5,4 \mathrm{mg} / \mathrm{kg} \\
\text { em bolus, seguida de } 4,0 \mathrm{mg} / \mathrm{kg} / \mathrm{h} \\
\text { durante } 23 \text { horas. } \\
\text { Grupo } 3(\mathrm{n}=171) \text { : administração do } \\
\text { placebo nos mesmos regimes } \\
\text { posológicos. }\end{array}$ & 10 & EUA \\
\hline Bracken $^{12}$ & 1997 & $\begin{array}{l}\text { Grupo } 24 \mathrm{MP}(\mathrm{n}=166) \text { : Metilprednisolona } \\
30 \mathrm{mg} / \mathrm{kg} \text { em bolus, seguido de } 5,4 \\
\mathrm{mg} / \mathrm{kg} / \mathrm{h} \text { durante } 24 \text { horas. } \\
\mathrm{Grupo} 48 \mathrm{MP}(\mathrm{n}=167): \text { Metilprednisolona } \\
30 \mathrm{mg} / \mathrm{kg} \text { em bolus, seguido de } 5,4 \\
\mathrm{mg} / \mathrm{kg} / \mathrm{h} \text { durante } 48 \text { horas. } \\
\text { Grupo } 48 \mathrm{MT}(\mathrm{n}=166): \text { Mesilato de } \\
\text { Tirilazad } 2,5 \mathrm{mg} / \mathrm{kg} \text { em bolus, a cada } 6 \\
\text { horas, por } 48 \text { horas. }\end{array}$ & 16 & EUA \\
\hline Bracken $^{13}$ & 1998 & $\begin{array}{l}\text { Grupo } 24 \mathrm{MP}(\mathrm{n}=145): \text { Metilprednisolona } \\
30 \mathrm{mg} / \mathrm{kg} \text { em bolus, seguido de } 5,4 \\
\mathrm{mg} / \mathrm{kg} / \mathrm{h} \text { durante } 24 \text { horas. } \\
\mathrm{Grupo} 48 \mathrm{MP}(\mathrm{n}=141): \text { Metilprednisolona } \\
30 \mathrm{mg} / \mathrm{kg} \text { em bolus, seguido de } 5,4 \\
\mathrm{mg} / \mathrm{kg} / \mathrm{h} \text { durante } 48 \text { horas. } \\
\text { Grupo } 48 \mathrm{MT}(\mathrm{n}=145): \text { Mesilato de } \\
\text { Tirilazad } 2,5 \mathrm{mg} / \mathrm{kg} \text { em bolus, a cada } 6 \\
\text { horas, por } 48 \text { horas. }\end{array}$ & 16 & EUA \\
\hline Pointillart ${ }^{14}$ & 2000 & $\begin{array}{l}\text { Grupo M }(n=27) \text { : Metilprednisolona } 30 \\
\mathrm{mg} / \mathrm{kg} \text { por } 1 \mathrm{~h} \text {, seguido por } 5,4 \mathrm{~m} / \mathrm{kg} / \mathrm{h} \\
\text { por } 23 \text { horas. } \\
\text { Grupo } N(\mathrm{n}=27) \text { : Nimodipina } 0,15 \\
\mathrm{mg} / \mathrm{kg} / \mathrm{h} \text { por } 2 \mathrm{~h} \text { seguido por } 0,03 \\
\mathrm{mg} / \mathrm{kg} / \mathrm{h} \text { for } 7 \text { dias. } \\
\text { Grupo } \mathrm{MN}(\mathrm{n}=27) \text { : Soma das } \\
\text { intervenções dos grupos } \mathrm{M} \text { e } \mathrm{N} \text {. } \\
\text { Grupo controle }(\mathrm{n}=25) \text { : sem intervenção; } \\
\text { aplicação de placebo. }\end{array}$ & 1 & França \\
\hline
\end{tabular}


Todos os estudos incluídos nesta revisão estão no nível um de evidência da classificação do Centro de Medicina Baseada em Evidências da Universidade de Oxford, na categoria para pesquisas sobre benefício de tratamento. Nos três estudos, 1092 pacientes foram randomizados e alocados na comparação entre a metilprednisolona, placebo, naloxona, mesilato de tirilazad e nimodipina.

$\mathrm{Na}$ avaliação da validade interna e risco de viés, apenas dois dos estudos incluídos (40\%), relativos ao third National Acute Spinal Cord Injure Study (NASCIS III), alcançaram pontuação suficiente para boa qualidade. Os critérios de mascaramento e descrição das perdas foram os que acarretaram maiores perdas de pontuação no score (Quadro $3)$.

Quadro 3. Análise de validade interna e risco de viés dos artigos analisados.

\begin{tabular}{|c|c|c|c|c|c|}
\hline \multirow{2}{*}{$\begin{array}{c}\text { Item do Score de } \\
\text { Jadad }\end{array}$} & \multicolumn{5}{|c|}{ Estudos } \\
\hline & Bracken $^{10}$ & Bracken $^{11}$ & Bracken $^{12}$ & Bracken $^{13}$ & Pointillart $^{14}$ \\
\hline $\begin{array}{l}\text { 1. O estudo foi } \\
\text { descrito como } \\
\text { randomizado? }\end{array}$ & $\operatorname{Sim}(1)$ & $\operatorname{Sim}(1)$ & $\operatorname{Sim}(1)$ & $\operatorname{Sim}(1)$ & $\operatorname{Sim}(1)$ \\
\hline $\begin{array}{l}\text { 2. O estudo foi duplo- } \\
\text { cego? }\end{array}$ & $\operatorname{Sim}(1)$ & $\operatorname{Sim}(1)$ & $\operatorname{Sim}(1)$ & $\operatorname{Sim}(1)$ & Não (0) \\
\hline $\begin{array}{l}\text { 3. Foram descritas as } \\
\text { perdas e exclusões? }\end{array}$ & Não (0) & Não (0) & $\operatorname{Sim}(1)$ & $\operatorname{Sim}(1)$ & $\operatorname{Sim}(1)$ \\
\hline $\begin{array}{l}\text { Soma parcial } 1 \\
\text { (SP1) }\end{array}$ & 2 & 2 & 3 & 3 & 2 \\
\hline $\begin{array}{l}\text { 4. A randomização foi } \\
\text { descrita e é } \\
\text { adequada? }\end{array}$ & $\operatorname{Sim}(+1)$ & $\operatorname{Sim}(+1)$ & $\operatorname{Sim}(+1)$ & $\operatorname{Sim}(+1)$ & $\operatorname{Sim}(+1)$ \\
\hline $\begin{array}{l}\text { 5. O mascaramento } \\
\text { foMedical Subject } \\
\text { Headingsi descrito e é } \\
\text { adequado? }\end{array}$ & Não $(-1)$ & Não $(-1)$ & Não $(-1)$ & Não $(-1)$ & Não $(-1)$ \\
\hline $\begin{array}{l}\text { Soma parcial } 2 \\
\text { (SP2) }\end{array}$ & $\mathbf{0}$ & $\mathbf{0}$ & $\mathbf{0}$ & $\mathbf{0}$ & $\mathbf{0}$ \\
\hline Score (SP1 + SP2) & 2 & 2 & 3 & 3 & 2 \\
\hline Interpretação & $\begin{array}{c}\text { Baixa } \\
\text { qualidade }\end{array}$ & $\begin{array}{c}\text { Baixa } \\
\text { qualidade }\end{array}$ & $\begin{array}{c}\text { Boa } \\
\text { qualidade }\end{array}$ & $\begin{array}{c}\text { Boa } \\
\text { qualidade }\end{array}$ & $\begin{array}{c}\text { Baixa } \\
\text { qualidade }\end{array}$ \\
\hline
\end{tabular}




\section{Resultados em curto e médio prazo de seguimento}

O segundo estudo nacional de lesões agudas da medula espinhal (NASCIS II) avaliou em curto prazo os desfechos dos pacientes com seis semanas e seis meses de seguimento. A administração de metilprednisolona proporcionou maior sobrevida em número absoluto, mas similar entre os grupos $(p=0,465)$. Na mensuração realizada com seis semanas de seguimento, apresentou ganho significativo no subgrupo de pacientes que receberam a metilprednisolona até oito horas após a lesão; houve melhores resultados de função motora $(p=0,048)$ e de sensibilidade do tato $(p=0,034)$, mas sem ganho na sensação de alfinete/picada $(p=0,061)$, ainda que a média tenha sido bem superior. A avaliação do mesmo subgrupo da naloxona também evidenciou valores médios de scores neurológicos superiores ao grupo controle, porém, sem diferença significante. Na avaliação com seis meses, os pacientes com metilprednisolona apresentaram melhores resultados de sensibilidade do tato $(p=0,042)$ e discriminação do alfinete $(p=0,012)$, em comparação com o placebo. Os resultados do subgrupo de pacientes com início de tratamento até oito horas de lesão foram significativamente melhores nos três desfechos primários supracitados. Sangramento gastrointestinal e infecções ocorreram em $7,1 \%$ e 4,5\%, respectivamente, dos pacientes que receberam metilprednisolona, sem diferença significante destes desfechos secundários de segurança entre os grupos $^{10}$. 
Inspirado nos resultados na análise de subgrupos do NASCIS II, desenvolveu-se o terceiro estudo nacional de lesões agudas da medula espinhal norte-americano (NASCIS II). Desta vez, a análise incluiu apenas pacientes que iniciaram o tratamento até oito horas após a lesão, com subgrupos de tratamento até três horas, entre três e oito horas e os menores de oito horas. Quanto à segurança dos esquemas, a sepse foi registrada em $2,6 \%$ dos pacientes do grupo que recebeu metilprednisolona por 48 horas, em comparação a $0,6 \%$ nos que receberam a droga por 24 horas, e nenhum no grupo do mesilato de tirilazad $(p=0,07)$, quando medido este desfecho em seis semanas de seguimento. Os pacientes com esquema de metilprednisolona de maior duração (48 horas) também concentrou mais casos de pneumonia grave $(p=0,02)$. A sobrevivência dos três grupos foi similar $(p=0,97)^{12}$.

Considerando $\circ$ grupo de tratamento com metilprednisolona por 24 horas como controle, o tratamento com mesilato de tirilazad não produziu scores motores maiores com significância estatística em nenhum dos cenários testados. Os pacientes tratados com metilprednisolona por 48 horas, subgrupo de até oito horas, tiveram melhor score motor, quando analisados os pacientes que cumpriram todo o protocolo da droga $(p=0,04)$. Nos pacientes que iniciaram tratamento em até três horas de injúria medular não houve ganhos significantes, e em todos os cenários de pacientes com tratamento entre três e oito horas a metilprednisolona causou ganhos de escore 
significantes. As avaliações da sensibilidade dos pacientes não tiveram seus resultados divulgados na íntegra, embora não tenham alcançado, em nenhum cenário, melhora significante, segundo relato dos pesquisadores. Não houve melhora significante na independência funcional nos grupos de intenção para tratar em nenhum dos momentos de avaliação, nem naqueles pacientes que completaram o protocolo do estudo, ainda que alguns domínios do índice, como autocuidado e controle de esfíncter, tenham apresentado melhores resultados no grupo de metilprednisolona por 48 horas $^{12}$.

\section{Resultados do seguimento em longo prazo}

O grupo de pacientes com naloxona apresentou maior sobrevida absoluta na avaliação de seguimento de um ano do segundo estudo nacional norte-americano, ainda que as curvas tenham sido similares $(p=0,525)$. Pacientes tratados com naloxona apresentaram menos episódios de pneumonia que o grupo com metilprednisolona (1,4\% vs. 3,3\%, $p=0,04)$. Mais uma vez, assim como no segmento até 180 dias, considerando-se os desfechos motores nos pacientes no geral tratados com metilprednisolona, não houve melhora significante. Para encontrar resultados significantes, foi realizada uma análise de subgrupo: estratificou-se os pacientes entre aqueles que iniciaram o tratamento em até oito horas e após este prazo. Em seguida, este primeiro subgrupo foi segregado por tipo de lesão medular. Apenas pacientes com perda sensorial completa abaixo da lesão 
conseguiram escores motores significantes, em um ano de seguimento. Quanto à avaliação do tato e discriminação da pontada de alfinete, não houve ganho com a metilprednisolona, em comparação com o placebo ${ }^{11}$.

No seguimento de um ano do terceiro estudo norteamericano, a sobrevivência entre os três grupos variou entre 92,8 a $94,6 \%(p=0,8)$. Quanto à avaliação motora, tanto no grupo de base quanto naqueles que completaram o esquema de tratamento do protocolo, não houve ganhos significantes nos pacientes tratados com metilprednisolona por 48 horas, em comparação com o grupo tratado por 24 horas, além de haver oscilação nos valores absolutos do braço de intervenção, com scores ora maiores, ora menores, em comparação ao grupo de controle. O mesmo cenário foi encontrado na avaliação sensorial e independência funcional ${ }^{13}$.

Dos 106 pacientes avaliados no estudo francês incluído nesta revisão, cinco morreram no seguimento de um ano, uma sobrevida de 95,2\%. Em todos os braços do estudo houve melhora significativa do score da avaliação motora, da sensação de picada de afinete e de tato durante 0 seguimento. Contudo, a diferença de pontuação entre os grupos não foi significante, inclusive, o braço de placebo apresentou pontuação maior em um ano de seguimento nas avaliações de picada de alfinete e tato. Apenas o tipo de lesão (completa ou incompleta) se correlacionou positivamente com a recuperação sublesional $(p<0,001)$. Quanto à segurança da droga, a metilprednisolona foi 
responsável por causar mais episódios de infecção do trato urinário, septicemia, doenças pulmonares, sangramento gastrointestinal e hipoglicemia, com resultado significante nesta última (16 vs. $1, p<0,05$ ). Os pacientes tratados com metilprednisolona necessitaram de menor permanência na Unidade de Terapia Intensiva (UTI) enquanto o braço de intervenção com metilprednisolona e nimodipina permaneceu menor período em ventilação mecânica invasiva, ambos não significantes ${ }^{14}$.

\section{DISCUSSÃO}

Esta revisão sumarizou os achados de cinco estudos na abordagem farmacológica do tratamento agudo do traumatismo da medula espinhal com metilprednisolona. Os trabalhos incluídos neste estudo variaram sua qualidade de baixa $(60 \%)$ a boa (40\%), e nenhum atingiu a categoria de mega trial. Os benefícios desta intervenção ficaram restritos a alguns subgrupos, ainda que alguns escores de avaliação motora tenham melhorado, não foram significantes.

O esforço para desenvolver uma terapêutica efetiva na neuroproteção remonta à década de 1970, nos Estados Unidos. Na década seguinte, foi lançado o primeiro estudo randomizado, de fase II, para avaliar a posologia desta droga, o primeiro estudo nacional estadunidense de injúria medular aguda (NASCIS I). Nos dois momentos iniciais de avaliação, não houve melhora na função motora $e$ sensibilidade. $O$ achado principal do estudo foi maior 
mortalidade precoce, complicações e efeitos adversos no grupo que recebeu altas doses da medicação ${ }^{15}$.

Mesmo diante dos achados do NASCIS $\mathrm{I}^{15,16}$, prosseguiram-se os testes de fase III no NASCIS II e NASCIS III, incluídos neste estudo, que não apresentaram ganho significante de função motora ou sensibilidade nos braços de intervenção: ganhos que até apareceram em curto prazo, ou em subgrupos, não se confirmaram em longo prazo. A análise de subgrupos, ainda que tendo sido especificada no relato do método dos estudos incluídos, foi um aspecto criticado nos anos subsequentes às publicações ${ }^{17}$ pois, ainda que os benefícios se aplicassem a grupos muito restritos de pacientes, a recomendação endossada aos protocolos clínicos e trazida nas conclusões dos estudos foi de uso irrestrito nos pacientes, o que não se sustenta, frente aos achados descritos nas publicações.

A recomendação da revisão da Colaboração Cochrane que discorre sobre o uso de fármacos esteroides no manejo do trauma raquimedular indica o uso da metilprednisolona em todos os pacientes traumatizados ${ }^{5}$, sem especificar a eficácia maior em apenas alguns subgrupos restritos. Contudo, foi um trabalho sistemático realizado por um único autor, que é o pesquisador principal dos três estudos nacionais norte-americanos (NASCIS I, II e III) sobre manejo agudo da injúria medular, acarretando em, pelo menos, conflito de interesses acadêmico.

Em contrapartida, não há recomendação do uso de metilprednisolona nos pacientes pediátricos com 
traumatismos da medula espinhal ${ }^{18}$. Frente à realidade de que os estudos desta revisão sistemática não incluíram crianças em suas análises, assume-se a recomendação de que o uso desta droga nesta faixa etária não deve ser considerada como uma opção de tratamento da injúria medular traumática ${ }^{18}$.

O uso rotineiro da metilprednisolona não deve ser seguido no traumatismo da medula espinhal, haja vista que não há demonstração de ganhos motores no seguimento de longo prazo, além de estudos de coorte apresentarem risco mais elevado de desenvolvimento de sangramento gastrointestinal ${ }^{19}$.

Uma coorte canadense comparou a aplicação do protocolo de metilprednisolona do segundo estudo nacional norte-americano ${ }^{10,11}$ numa proporção de $1: 1$. Não houve mortalidade registrada em nenhum dos grupos e, corroborando os achados dos braços de intervenção, não houve melhora motora, bem como as complicações foram maiores no grupo que houve a administração da droga $(p<0,02)^{20}$.

Responsáveis por mudar o critério de atendimento emergencial dos lesados medulares no final dos anos 1990 e início dos anos 2000, os estudos NASCIS II e NASCIS III apresentavam ao longo dos quatro textos incluídos neste estudo $^{10-13}$ diversas afirmativas que remetiam informações com ganho absoluto em alguns scores de avaliação motora ou de sensibilidade, sem que, contudo, houvesse garantia estatística de que ele não se dava por um acaso. Cabe 
ressaltar ainda que, os ganhos de dois ou três pontos não chegaram a ser clinicamente significantes frente à amplitude das escalas utilizadas que podiam chegar a 70 unidades. Assim, não houve melhora nas medidas dos desfechos primários avaliados, pontuando a evidência de uso do medicamento como fraca, a despeito da boa elaboração e condução do NASCIS II e III $^{21}$.

Ainda que o foco desta revisão seja os resultados que concernem ao braço de intervenção com metilprednisolona dos estudos, as demais drogas comparadas não mostraram benefício frente aos grupos controle. Alguns estudos preliminares atuais apontaram modestos benefícios neuroprotetivos do uso de vitamina D associada a eritropoietina e progesterona, ainda que sejam necessários estudos mais robustos e bem delineados, para que possa ser realizada uma recomendação de uso clínico ${ }^{22}$.

Poucos estudos relataram custos hospitalares decorrentes do uso desta intervenção, mas uma metanálise que avaliou este desfecho pôde apresentar dados significativos de que a metilprednisolona reduz os dias de cuidados gerais e o período de reabilitação, mas eleva o tempo de ventilação mecânica e de permanência na unidade de terapia intensiva. Os custos gerais, no entanto, não diferem entre ambos os braços ${ }^{23}$.

O uso da metilprednisolona leva a incremento significativo do risco de hipoglicemia e pneumonia. Sangramento gastrointestinal, sepse, úlceras de decúbito, infecções do trato urinário, ainda que figurando 
significativamente em estudos isolados com risco elevado, não se sustentam no cenário da avaliação global dos achados $^{24}$.

Suporte para o desenho dos estudos NASCIS, os resultados de pesquisa de bancada favoráveis ao uso de metilprednisolona não se concretizaram, quando avaliados sob uma metanálise: $34 \%$ dos estudos apresentaram melhora nos desfechos enquanto $58 \%$ tiveram resultados de que a intervenção não melhora os dados das avaliações ${ }^{25}$. Além das limitações intrínsecas destes tipos de estudos e da dificuldade de reprodutibilidade, a heterogeneidade de animais utilizados como cobaias, aliada à diversidade de métodos aplicados, reduz a recomendação de seus achados.

Diante da falha de opções farmacológicas, as estratégias atuais de controle da lesão medular aguda podem compreender, cirurgicamente, a descompressão e fixação cirúrgica precoce, associada ao uso de fármacos vasopressores para melhorar a perfusão da medula espinhal. Norepinefrina e cuidados neurocríticos, com a assistência de monitoramento invasivo, apresentam-se incipientes na busca por estratégias efetivas que melhorem o tratamento de suporte aos pacientes. Além de metilpredinisolona, nimodipina, naloxona e mesilato de tirilazad - drogas testadas nos estudos incluídos -, outras drogas, como gliburida, substâncias autólogas, como o fator básico de crescimento de fibroblastos, e intervenções, a exemplo de estimulação elétrica funcional, também não mostraram benefícios quando testados em ensaios clínicos ${ }^{26}$. 
A avaliação da qualidade de vida, um desfecho substitutivo de valor secundário nesta pesquisa, não foi realizada em nenhum dos estudos incluídos. A independência funcional, quando mensurada, não melhorou significativamente com a intervenção da metilprednisolona. Diante da dificuldade dos pacientes em realizar a adaptação após o evento traumático, as intervenções no traumatismo de medula espinhal também devem ser capazes de contribuir com melhores resultados da qualidade de vida 27.

Em suma, o uso global de metilprednisolona em baixas doses estabelecidas no NASCIS I e testadas nos estudos NASCIS II e III, bem como no estudo francês, não melhora os desfechos motores e de sensibilidade dos pacientes, com elevação substancial de efeitos colaterais, devendo não ser administrada (nível de evidência: I; força da recomendação: forte).

\section{CONCLUSÃO}

Diante das evidências sumarizadas e de sua análise crítica, não recomendamos o uso de metilprednisolona no tratamento do traumatismo da medula espinhal, face a ausência de benefício de melhora significativa da função motora e sensibilidade, além de não impactar na mortalidade. Quanto aos desfechos substitutivos secundários, a independência funcional também não apresentou melhora, enquanto a qualidade de vida sequer foi avaliada nos estudos incluídos. O uso da droga também se associou estatisticamente à elevação de efeitos adversos. 


\section{REFERÊNCIAS}

1. Hachem LD, Ahuja CS, Fehlings MG. Assessment and management of acute spinal cord injury: From point of injury to rehabilitation. J Spinal Cord Med 2017;40:665-75. http://dx.doi.org/10.1080/10790268.2017.1329076

2.Ghajarzadeh M, Rahimi FA, Nedjat S, Sheikhrezaei A, Saberi H. Survival Analysis in Patients with Chronic Traumatic Spinal Cord Injury. Iran J Public Health 2019;48:2260-9.

https://www.ncbi.nlm.nih.gov/pmc/articles/PMC6974852/pdf/IJPH48-2260.pdf

3.Jain NB, Ayers GD, Peterson EN, Harris MB, Morse L, O'Connor KC, et al. Traumatic spinal cord injury in the United States, 1993-2012. JAMA 2015;313:2236-43. http://dx.doi.org/10.1001/jama.2015.6250 4.Del Bel EA, Silva CA, Mladinic M. O trauma raquimedular. Coluna/Columna 2009;8:441-9. $\quad$ https://doi.org/10.1590/S180818512009000400017

5.Bracken MB. Steroids for acute spinal cord injury. Cochr Datab Syst Rev 2012;1(CD001046):1-40.

http://dx.doi.org/10.1002/14651858.CD001046.pub2

6. Ministério da Saúde (Brasil). Diretrizes metodológicas: elaboração de revisão sistemática e metanálise de ensaios clínicos randomizados. Brasília: Ministério da Saúde; 2012.

https://bvsms.saude.gov.br/bvs/publicacoes/diretrizes metodologica s elaboracao sistematica.pdf

7.Galvão TF, Pansani TSA, Harrad D. Principais itens para relatar Revisões sistemáticas e Meta-análises: A recomendação PRISMA. Epidemiol Serv Saúde 2015;24:335-42. http://dx.doi.org/10.5123/S1679-49742015000200017

8.Jadad AR, Moore RA, Carroll D, Jenkinson C, Reynolds DJ, Gavaghan $D J$, et al. Assessing the quality of reports of randomized clinical trials: is blinding necessary? Control Clin Trials 1996;17:1-12. http://dx.doi.org/10.1016/0197-2456(95)00134-4

9.Oxford Centre for Evidence-Based Medicine. The Oxford Levels of Evidence 2. (endereço na Internet. London: University of Oxford, Centre for Evidence-Based Medicine. (Acessado em 16/05/2020). Disponível em: https://www.cebm.net/index.aspx?o=5653

10.Bracken MB, Shepard MJ, Collins WF, Holford TR, Young W, Baskin DS, et al. A Randomized, Controlled Trial of Methylprednisolone or Naloxone in the Treatment of Acute Spinal-Cord Injury. Results of the Second National Acute Spinal Cord Injury Study. N Engl J Med 1990;322:1405-11.

http://dx.doi.org/10.1056/NEJM199005173222001

11.Bracken MB, Shepard MJ, Collins WF, Holford TR, Baskin DS, Eisenberg HM, et al. Methylprednisolone or Naloxone Treatment After Acute Spinal Cord Injury: 1-year Follow-Up Data. Results of the Second National Acute Spinal Cord Injury Study. J Neurosurg 1992;76:23-31. http://dx.doi.org/10.3171/jns.1992.76.1.0023

12.Bracken MB, Shepard MJ, Holford TR, Leo-Summers L, Aldrich EF, Fazl M, et al. Administration of Methylprednisolone for 24 or 48 Hours 
or Tirilazad Mesylate for 48 Hours in the Treatment of Acute Spinal Cord Injury. Results of the Third National Acute Spinal Cord Injury Randomized Controlled Trial. National Acute Spinal Cord Injury Study. JAMA 1997;277:1597-604.

http://dx.doi.org/10.1001/jama.1997.03540440031029

13.Bracken MB, Shepard MJ, Holford TR, Leo-Summers L, Aldrich EF, Fazl M, et al. Methylprednisolone or Tirilazad Mesylate Administration After Acute Spinal Cord Injury: 1-year Follow Up. Results of the Third National Acute Spinal Cord Injury Randomized Controlled Trial. J Neurosurg 1998;89:699-706.

http://dx.doi.org/10.3171/jns.1998.89.5.0699

14. Pointllart V, Petitjean ME, Wiart L, Vital JM, Lassié P, Thicoipé M, et al. Pharmacological Therapy of Spinal Cord Injury During the Acute Phase. Spinal Cord 2000;38:71-6.

http://dx.doi.org/10.1038/sj.sc.3100962

15.Bracken MB, Collins WF, Freeman DF, Shepard MJ, Wagner FJ, Silten RM, et al. Efficacy of Methylprednisolone in Acute Spinal Cord Injury. JAMA 1984;251:45-52.

http://dx.doi.org/10.1001/jama.1984.03340250025015

16.Bracken MB, Shepard MJ, Hellenbrand KG, Collins WF, Leo LS, Freeman DF, et al. Methylprednisolone and Neurological Function 1 Year After Spinal Cord Injury. Results of the National Acute Spinal Cord Injury Study. J Neurosurg 1985;63:704-13.

http://dx.doi.org/10.3171/jns.1985.63.5.0704

17. Hextrum S, Bennet S. A Critical examination of Subgroup Analyses: The national Acute Spinal Cord injury Studies and Beyond. Front Neurol 2018;9:1-3. http://dx.doi.org/10.3389/fneur.2018.00011

18.Caruso MC, Daugherty MC, Moody SM, Falcone RA, Bierbrauer KS, Geis GL. Lessons Learned From Administration of High-Dose Methylprednisolone Sodium Succinate for Acute Pediatric Spinal Cord Injuries. J Neurosurg Pediatr 2017;20:567-74. http://dx.doi.org/10.3171/2017.7.PEDS1756

19.Evaniew N, Belley-Côté EP, Fallah N, Noonan VK, Rivers CS, Dvorak MF. Methylprednisolone for the Treatment of Patients with Acute Spinal Cord Injuries: A Systematic Review and Meta-Analysis. J Neurotrauma 2016;33:468-81. http://dx.doi.org/10.1089/neu.2015.4192

20.Evaniew N, Noonan VK, Fallah N, Kwon BK, Rivers CS, Ahn H, et al. Methylprednisolone for the Treatment of Patients with Acute Spinal Cord Injuries: A Propensity Score-Matched Cohort Study from a Canadian Multi-Center Spinal Cord Injury Registry. J Neurotrauma 2015;32:1674-83. http://dx.doi.org/10.1089/neu.2015.3963

21. Hurlbert RJ. Methylprednisolone for acute spinal cord injury: an inappropriate standard of care. J Neurosurg 2000;93(1 Suppl):1-7. http://dx.doi.org/10.3171/spi.2000.93.1.0001

22.Joaquim AF, Daniel JW, Schroeder GD, Vaccaro AR. Neuroprotective Agents as an Adjuvant Treatment in Patients With Acute Spinal Cord Injuries: A Qualitative Systematic Review of Randomized Trials. Clin Spine Surg 2020;33:65-75.

http://dx.doi.org/10.1097/BSD.0000000000000861 
23. Liu Z, Yang $Y$, He L, Pang M, Luo C, Liu B, et al. High-dose methylprednisolone for acute traumatic spinal cord injury: A metaanalysis. Neurology 2019;93:e841-50.

http://dx.doi.org/10.1212/WNL.0000000000007998

24.Sultan I, Lamba N, Liew A, Doung P, Tewarie I, Amamoo JJ, et al. The safety and efficacy of steroid treatment for acute spinal cord injury: A Systematic Review and meta-analysis. Heliyon 2020;6:e03414. http://dx.doi.org/10.1016/j.heliyon.2020.e03414 25.Akhtar AZ, Pippin JJ, Sandusky CB. Animal studies in spinal cord injury: a systematic review of methylprednisolone. Altern Lab Anim 2009;37:43-62. http://dx.doi.org/10.1177/026119290903700108

26. Karsy M, Hawryluk G. Modern Medical Management of Spinal Cord Injury. Curr Neurol Neurosci Rep 2019;19:65. http://dx.doi.org/10.1007/s11910-019-0984-1

27. Silveira SL, Ledoux TA, Johnston CA, Kalpakjian C, O'Connor DP, Cottingham $\mathrm{M}$, et al. Differences among participants' response to a wellness intervention for individuals with spinal cord injuries. Disabil Health J 2020;13:100842.

http://dx.doi.org/10.1016/j.dhjo.2019.100842 